\title{
Efficacy and Safety of Single-Session Radiofrequency Ablation in Treating Benign Thyroid Nodules: A Short-Term Prospective Cohort Study
}

\author{
Van Bang Nguyen $\mathbb{D}^{1},{ }^{1}$ Thi Xuan Nguyen, ${ }^{1}$ Van Vy Hau Nguyen, ${ }^{1}$ Hai Thuy Nguyen, ${ }^{2}$ \\ Dinh Toan Nguyen, ${ }^{2}$ and Chi Van Le $\mathbb{B}^{2}$ \\ ${ }^{1}$ Center of Endocrinology and Diabetes, Family Hospital, Da Nang, Vietnam \\ ${ }^{2}$ Department of Internal Medicine, Hue University of Medicine and Pharmacy, Hue University, Hue City, Vietnam \\ Correspondence should be addressed to Chi Van Le; lvanchi@hueuni.edu.vn
}

Received 25 June 2021; Revised 15 August 2021; Accepted 2 September 2021; Published 13 September 2021

Academic Editor: Thomas J. Fahey

Copyright (c) 2021 Van Bang Nguyen et al. This is an open access article distributed under the Creative Commons Attribution License, which permits unrestricted use, distribution, and reproduction in any medium, provided the original work is properly cited.

\begin{abstract}
Objective. The aims of this study are to evaluate the safety and efficacy of RFA in the treatment of benign thyroid nodule(s) and to find independent factors related to the volume reduction rate of the nodule(s). Materials and Methods. This short-term prospective study from a single medical center was conducted on 93 benign thyroid nodules in 93 patients treated with RFA. Two basic techniques were used: the trans-isthmic approach and moving-shot technique. Clinical and ultrasonography examinations were performed at 1- and 3-month follow-up after the treatment session. Primary outcomes included volume reduction ratio (VRR) at 1-month and 3-month follow-ups; secondary outcomes were therapeutic success rate and complications. Multiple linear regression analysis was used to determine independent factors associated with VRR. Results. A final sample of 78 patients with 78 nodules, given participant rate $83.8 \%$ (including 60 solid nodules, 16 predominantly cystic nodules, and 2 thyroid cysts), was followed up for 3 months. The mean volume reduction ratio was $41.47 \%$ and $64.72 \%$ after 1 -month and 3 -month follow-ups, respectively. The therapeutic success rate was $30.8 \%$ at 1 -month and $84.6 \%$ at 3 -month follow-ups. Symptom score and cosmetic score improved significantly. There was no change in thyroid function tests. Two minor complications (transient voice change) were found. The multiple linear regression analysis showed that the internal component of the nodules significantly related to the VRR during the 3-month follow-up ( $\beta=23.00$; 95\%CI (7.59-38.45)). Conclusion. RFA was demonstrated as a safe and effective option for benign thyroid nodules treatment. It can be used as an alternative treatment with encouraging results.
\end{abstract}

\section{Introduction}

Thyroid nodule(s) is the second most common endocrine disorder after diabetes, its prevalence being largely dependent on the examination method of high-frequency ultrasound, which can be detected in $20-76 \%$ of the adults $[1,2]$. Once the benign nodule has been confirmed, ongoing treatment usually depends on the onset of nodule-related compressible and/or cosmetic symptoms [3, 4]. In Vietnam and worldwide, surgery or T4-suppressive therapy is preferred for treating thyroid nodule(s), but both have limitations. Drawbacks of surgery are general anesthesia, iatrogenic hypothyroidism, and scarring of the neck [5].
Using T4-suppressive therapy is controversial and associated with the high risks of iatrogenic hyperthyroidism, decreased bone density, atrial fibrillation, and increasing overall morbidity and mortality from cardiovascular diseases $[6,7]$.

In recent years, radiofrequency ablation (RFA) has been emerging from one of the treatments of benign thyroid nodule(s) as a minimally invasive alternative treatment with encouraging results, fewer complications than surgery, high feasibility of performing in an outpatient room, and preservation of thyroid function [8-10]. It can induce thyroid tissue heating and necrosis, and nodule volume will be shrunk afterward, which in turn alleviates the associated 
symptoms or cosmetic issue [11]. Theoretically, RFA usually used 2 basic techniques including the moving-shot technique and the trans-isthmic approach with the guidance of ultrasonography (US) [12]. Factors related to the efficacy of this therapy in benign thyroid nodule(s) treatment have been reported in numerous studies in the world including Vietnam. It included initial volume, US features, vascularity grade, and initial ablation ratio (IAR) [13-18]. However, conclusions are still debated. Therefore, this short-term prospective study from a single medical center set out to evaluate the safety and efficacy of RFA in the treatment of benign thyroid nodule(s) and to find independent factors related to the volume reduction rate of the nodule(s).

\section{Methods}

2.1. Study Design and Patient Selection. This prospective cohort single-center study was approved by the Ethics Committee of the Institutional Review Board of Danang Family Hospital, Danang, Vietnam (number: 12.04-30218), and written informed consent for procedures was obtained for all patients.

All patients were selected with the following inclusion criteria: (1) benign thyroid nodule(s) was confirmed by following recommendations from the Asian Conference on Tumor Ablation Task Force: US result and at least two separate US-guided fine-needle aspirations (US-FNAs) cytology or US-guided core needle biopsy (US-CNB) [19]; (2) reports of pressure symptoms (including pain, compressive symptoms, and neck discomfort) or cosmetic problems or anxiety about tumor; and (3) refusal to undergo surgery. The exclusion criteria were as follows: (1) the largest dimension of the nodule(s) less than $15 \mathrm{~mm}$; (2) nodule(s) showing established or suspected malignant features during US (according to ACR-TIRADS 4 to 5) or cytology (according to Bethesda class III to VI); (3) current thyrotoxicosis; (4) patients with short life expectancy by comorbidity of severe diseases; (5) pregnancy; and (6) patients lost to follow-up.

From January 2019 to October 2020, 93 patients who underwent treatment using the RFA were enrolled in this study.

\subsection{Measurement and Assessment}

2.2.1. Pretreatment Assessment and Radiofrequency Ablation Procedure. Before the procedure, conventional clinical examination, US, 2 times separated US-guided FNAs or CNB, and the laboratory test was performed. At registration, patients were evaluated for the compression symptoms using a $10 \mathrm{~cm}$ visual analog scale (0-10). An endocrinologist examined a cosmetic grade: 1 , no palpable mass; 2 , a palpable mass but no cosmetic problem; 3, cosmetic problem on swallowing only; and 4, readily detected cosmetic problem $[4,19,20]$. An 8 to $12 \mathrm{MHz}$ linear probe of a real-time ultrasound system (Acuson NX2 or NX3 series, Siemens Healthineer) was performed by only one radiologist with more than 5 years of experience. Nodule(s) was evaluated for the position, size, volume, solid/cystic proportions, echogenicity, and volume $(V=\pi a b c / 6$, where $a, b$, and $c$ are the 3 diameters). Ultrasound-guided FNA or CNB examinations were performed by a licensed endocrinologist with more than 3 years of experience (Nguyen VB). Thyroid function (thyroid-stimulating hormone (TSH) and free thyroxine (FT4) level) was obtained. Before the treatment of each patient, we explained the advantages and disadvantages of thyroid RFA.

All the procedures were performed by the same endocrinologist (Nguyen VB), who has a licensed certificate and more than 3 years of experience in US-FNA/CNB, thyroid ethanol ablation, and thyroid RFA, at the outpatient department of the Center of Endocrinology and Diabetes, Danang Family Hospital, Vietnam. In the RFA procedure, patients in a supine position with mild neck extension were performed skin sterilization and given local anesthesia with $2 \%$ lidocaine at the needle-puncture site. We used 18-gauge internally cooled monopolar electrodes ( $5 \mathrm{~mm}$ or $7 \mathrm{~mm}$ in active tips) which were connected to a radiofrequency generator (CoATherm AKF200, APRO KOREA Inc.) to puncture into the nodule under US guidance via the trans-isthmic approach (Figure 1(a)). The nodules were ablated by using the moving-shot technique (Figure 1(b)). Hydrodissection was used in a few cases to protect important structures such as the nerve and artery by slowly injecting $5 \%$ dextrose (Figure 1(c)). The transient hyperechoic zone proved nodules ablated completely $[12,19,20]$. In the case of the cystic nodule(s) or predominantly cystic nodule(s), fluid aspiration was performed completely before ablating the nodule and its vascularity. Patients were asked to stay in the hospital for 60 minutes after the procedure and discharged if having no complications.

2.3. Follow-Up of the Patients. Follow-up was performed at 1 and 3 months after the treatment section. In the first month after ablation, US evaluation, thyroid function tests (TSH and FT4), symptom score, and cosmetic score were evaluated. In other follow-ups, only the US examination was used. Volume reduction rate (VRR) of the treated nodule was calculated based on the following formula:

$$
\mathrm{VRR}=\frac{(\text { Baseline volume }- \text { posttreatment volume })}{\text { Baseline volume }} \times 100 \% \text {. }
$$

A $>50 \%$ volume reduction of the initial nodule volume measured at each follow-up US examination was considered as a therapeutic success [16]. Also, we recorded any specific complaints or concerns in the follow-up period.

2.4. Efficacy Outcome. The primary endpoints were efficacy 1 month and 3 months after ablation through VRR. Therapeutic success was defined as a VRR $>50 \%$ of the initial nodule volume measured at each follow-up US examination [16]. Secondary endpoints were improvements in symptoms and cosmetic scores and no change of thyroid function tests. 


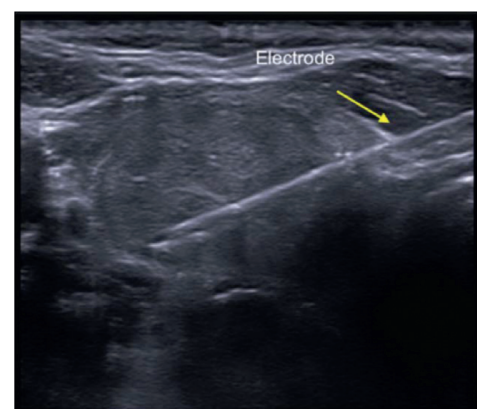

(a)

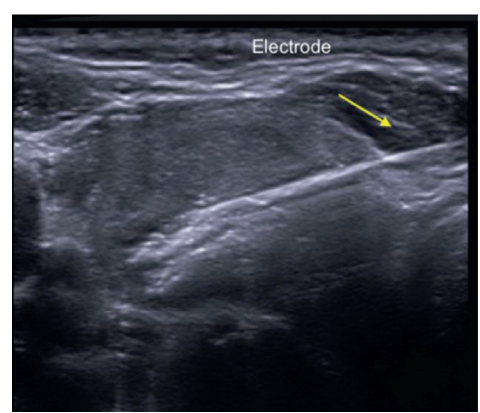

(b)

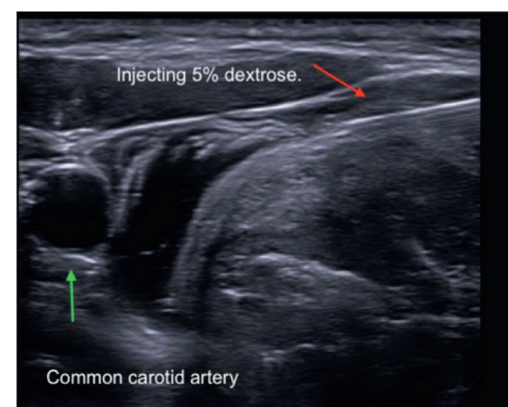

(c)

FIGURE 1: A 37-year-old woman presented with neck bulging. (a) Axial sonographic image shows the trans-isthmic approach of 5 mm size active tip of the electrode (yellow arrow). (b) Axial sonographic image shows the moving-shot technique (yellow arrow). After ablation of the peripheral imaginary unit, the electrode is moved to the central portion and ablation is performed. (c) The hydrodissection technique was applied to preventing important structures by injecting slowly $10 \mathrm{ml} 5 \%$ dextrose (red arrow).

2.5. Safety Outcome. Safety outcome (complications and side effects) followed that reported by the international working group on image-guided tumor ablation [21]. Major complications include substantial morbidity and disability which increases the level of care, hospital admission, hemorrhage needing a blood transfusion, and permanent voice change. Other complications were identified as minor complications (pain, transient voice change, vomiting, and skin burns).

2.6. Demographic Characteristics and Other Factors. In this study, demographic information included age (continuous variable) and sex (categorical variable: male and female). Treatment characteristics included ablation time (continuous variable: minute), max RF power (continuous variable: Watt), min RF power (continuous variable: Watt), and volume of lidocaine used (continuous variable: $\mathrm{ml}$ ).

2.7. Statistical Analysis. SPSS version 20.0 for Windows was used for all statistical analyses. The number of events and their percentage were calculated as the safety outcome. To evaluate the RFA efficacy, which is a numeric outcome variable, our purpose is to calculate the mean and standard deviation (SD) of the VRR during the follow-up period (1 month and 3 months after ablation). Although the scheduled examinations ( 1 month and 3 months after section treatment) were informed to patients, the included patients did not exactly follow this schedule due to individual reasons and COVID-19, as a limitation of this study. A general linear model with 3 times repeated measurement was used to compare changes in nodule volume, largest diameter, symptom score, and cosmetic score from the initial time to 1 month, 3 months after the procedure. The Friedman test or Wilcoxon's matched-pair signed-rank test was used to alternating paired $t$-tests to compare changes in volume reduction rate and therapeutic success rate from 1 month to 3 months after RFA if data cannot be assumed to be normally distributed. To identify factors that were independently predictive of efficacy (the volume reduction ratio at 3 months), we used multiple linear regression analysis.
Variables entered into the model included age (continuous variable), sex (categorical variable: male and female), symptom score (continuous variable: range from 1-10 score), cosmetic score (continuous variable: range from 1-4 score), initial volume (continuous variable), thyroid functions (TSH and FT4- continuous variable), characteristic of nodules (categorical variable: solid nodules, predominantly solid nodules, and cyst), ablation time (continuous variable), max RF power (continuous variable), min RF power (continuous variable), largest diameter (continuous variable), and volume of lidocaine used (continuous variable). Statistical significance was defined when the $P$ value was less than 0.05 .

\section{Results}

From January 2019 to October 2020, 93 patients who had undergone treatment of thyroid nodules using the RFA were enrolled in this study. Following treatment, 15 patients were lost before the 3 months follow-up. A final sample of 78 patients with 78 nodules, given participant rate $83.8 \%$, was followed up for 1 month and 3 months.

\subsection{Baseline Characteristics of the Patients and Nodules.} Table 1 shows the baseline characteristics of the patients and nodules. Most of the patients were female (89.3\%). The mean age was $44.82 \pm 14.72$ years (range 20-76). The mean symptom score and cosmetic score are 7.33 and 3.10 , respectively. On initial examination, the average largest diameter of the thyroid nodules was $27.20 \pm 8.74 \mathrm{~mm}$ (range 12.2-49.2) with a mean volume of $6.11 \pm 5.46 \mathrm{ml}$ (range 0.68-27.30). Among the 78 treated thyroid nodules, there were 60 solid $(76.9 \%), 16$ predominantly solid nodules (20.5\%), and $2(2.6 \%)$ cystic nodules. Thyroid functions include that mean concentrations of TSH and FT4 are $1.25 \mathrm{mUI} / \mathrm{ml}$ and $1.32 \mathrm{ng} / \mathrm{dL}$, respectively.

\subsection{Characteristics of Nodule Treatment and Safety Outcome.} Our treatment characteristics and safety outcomes are summarized in Table 2 . The mean volume of lidocaine $2 \%$ was $12.94 \mathrm{ml}$ (range $4-27 \mathrm{ml}$ ). Min and max of RF power 
TABLE 1: Baseline characteristics of the patients and nodules.

\begin{tabular}{|c|c|}
\hline Characteristics & Summary statistics $(N=78)$ \\
\hline Number of patients & 78 \\
\hline Number of nodules & 78 \\
\hline Age (years) $(($ mean $\pm S D)($ range $))$ & $44.82 \pm 14.72(20-76)$ \\
\hline Female $(n(\%))$ & $70(89.3)$ \\
\hline \multicolumn{2}{|l|}{ Nodule position $(n(\%))$} \\
\hline Left & $36(46.2)$ \\
\hline Isthmus & $0(0)$ \\
\hline Right & $42(53.8)$ \\
\hline Mean nodule volume $(\mathrm{ml})(($ mean $\pm \mathrm{SD})($ range $))$ & $6.11 \pm 5.46(0.68-27.30)$ \\
\hline Mean largest nodule diameter $(\mathrm{mm})(($ mean $\pm \mathrm{SD})($ range $))$ & $27.20 \pm 8.74(12.2-49.2)$ \\
\hline \multicolumn{2}{|l|}{ Internal nodule component $(n(\%))$} \\
\hline Solid & $60(76.9)$ \\
\hline Mix solid & $16(20.5)$ \\
\hline Cyst & $2(2.6)$ \\
\hline FT4 $(\mathrm{ng} / \mathrm{dL})(($ mean $\pm \mathrm{SD})($ range $))$ & $1.32 \pm 0.29(0.91-2.15)$ \\
\hline $\mathrm{TSH}(\mathrm{mIU} / \mathrm{ml})(($ mean $\pm \mathrm{SD})($ range $))$ & $1.25 \pm 0.79(0.01-4.25)$ \\
\hline Cosmetic score $(($ mean $\pm \mathrm{SD})$ (range) $)$ & $3.10 \pm 1.02(1-4)$ \\
\hline Symptom score $(($ mean \pm SD) (range) $)$ & $7.33 \pm 2.66(0-10)$ \\
\hline
\end{tabular}

TABLE 2: Characteristics of nodule treatment and safety outcome.

\begin{tabular}{lr}
\hline Characteristics & Summary statistics $(N=78)$ \\
\hline Lidocaine 2\% (ml) ((mean \pm SD) (range)) & $12.94 \pm 4.57(4-27)$ \\
Min RF power (Watt) ((mean \pm SD) (range) & $16.41 \pm 3.02(10-25)$ \\
Max RF power (Watt) ((mean \pm SD) (range)) & $31.02 \pm 8.28(20-45)$ \\
Ablation time (minute) ((mean \pm SD) (range) & $21.97 \pm 13.45(6.5-62)$ \\
Complication $(n(\%))$ & $2(2.56)$ \\
$\quad$ Minor complication & $0(0)$ \\
Major complication & \\
\hline
\end{tabular}

were 16.41 Watt and 31.02 Watt, respectively. The mean ablation time was 21.97 minutes (range 6.5-62 minutes). There were only two cases with minor complication (2.56\%) which was transient voice change and completely recovered one hour after an injection of cold $5 \%$ dextrose $\left(0^{\circ} \mathrm{C}\right.$ to $\left.4^{\circ} \mathrm{C}\right)$. There was no major complication.

3.3. The Treatment Outcome and Its Related Factors. The treatment outcomes are summarized in Table 3. The mean largest diameter of nodules decreased significantly from $27.20 \pm 8.74 \mathrm{~mm}$ at an initial time to $22.17 \pm 8.00 \mathrm{~mm}$ at 1 month after ablation and $18.50 \pm 6.86 \mathrm{~mm}$ at 3 months after ablation with $P<0.0001$. Mean nodule volume was $6.11 \pm 5.46 \mathrm{ml}, 3.55 \pm 3.34 \mathrm{ml}$, and $2.07 \pm 1.93$ at the initial time, 1-month, and 3-month follow-ups, respectively $(P<0.0001)$. This represents approximately $41.47 \%$ and $64.72 \%$ of the volume reduction ratio after 1 -month and 3 months follow-ups. The therapeutic success rate was $30.8 \%$ at 1 -month and $84.6 \%$ at 3 -month follow-ups. At the 3month follow-up, symptom score and cosmetic score decreased significantly from 7.33 to 3.79 (symptom score) and from 3.10 to 1.56 (cosmetic score) with $P<0.0001$. However, there was no change in thyroid function tests.

Table 4 summarizes the results of the multiple linear regression analysis, which revealed that the internal component of the nodules $(P<0.05)$ was independent factors that predicted the volume reduction rate at 3 months after ablation $(\beta=23.00 ; 95 \%$ CI $(7.59-38.45))$. All other clinical and US characteristics including age, sex, initial volume, TSH, FT4, cosmetic score, symptom score, ablation time, volume lidocaine $2 \%$, RF powers, and largest diameter did not show significant relations to the VRR at 3 months after ablation.

\section{Discussion}

The efficacy and safety of RF ablation were demonstrated from our prospective cohort single-center study which was performed by only one endocrinologist with 3 years of experience. Only two cases $(2.56 \%)$ with transient voice change occurred in 78 patients in 2 years of study, and no major complication was found. Thyroid functions were constant 1 month after ablation. Regarding efficacy, RFA reduced nodule volume by $41.47 \%$ and $64.72 \% 1$ and 3 months after ablation, respectively. This represents approximately $30.8 \%$ and $84.6 \%$ therapeutic success rates in this follow-up period. In addition, patients who were treated by RFA improved their symptom score and cosmetic score significantly. We found that only internal component of the nodules significantly related to the VRR during the 3-month follow-up $(\beta=23.00 ; 95 \% \mathrm{CI}(7.59-38.45))$.

Benign thyroid nodule(s) is a relatively common disease. Its traditional treatment options include surgery and T4-suppressive therapy. However, both of them had 
TABLE 3: Outcomes for 78 benign thyroid nodules after RF ablation

\begin{tabular}{|c|c|c|c|c|}
\hline Variables & Before & 1 month & 3 months & $P$ \\
\hline Largest diameter $(\mathrm{mm})$ & $27.20 \pm 8.74$ & $22.17 \pm 8.00$ & $18.50 \pm 6.86$ & 0.0001 \\
\hline Volume $(\mathrm{ml})($ mean $\pm \mathrm{SD})$ & $6.11 \pm 5.46$ & $3.55 \pm 3.34$ & $2.07 \pm 1.93$ & 0.0001 \\
\hline Volume reduction rate (\%) & & $41.47 \pm 19.92$ & $64.72 \pm 17.71$ & 0.0001 \\
\hline Symptom score & $7.33 \pm 2.65$ & $4.74 \pm 2.31$ & $3.79 \pm 1.64$ & 0.0001 \\
\hline Cosmetic score & $3.10 \pm 1.02$ & $1.64 \pm 0.93$ & $1.56 \pm 0.82$ & 0.0001 \\
\hline Therapeutic success $(n(\%))$ & & $24(30.8)$ & $66(84.6)$ & 0.0001 \\
\hline $\mathrm{TSH}(\mathrm{mIU} / \mathrm{ml})($ mean \pm SD $)$ & $1.25 \pm 0.79$ & $1.32 \pm 1.07$ & & 0.711 \\
\hline FT4 $(\mathrm{ng} / \mathrm{dL})($ mean $\pm \mathrm{SD})$ & $1.31 \pm 0.29$ & $1.28 \pm 0.27$ & & 0.505 \\
\hline
\end{tabular}

TABLE 4: Factors independently predictive of volume reduction via multiple linear regression analysis.

\begin{tabular}{lcccc}
\hline \multirow{2}{*}{ Variable } & \multicolumn{5}{c}{$95 \%$ CI of B } \\
& B & Lower & Upper & \\
\hline (Constant) & 9.03 & -52.41 & 70.47 & 0.76 \\
Age & 0.29 & -0.1 & 0.69 & 0.135 \\
Sex & -16.09 & -39.59 & 7.41 & 0.17 \\
Internal component of the & 23.00 & 7.59 & 38.45 & $\mathbf{0 . 0 0 5}$ \\
nodule & -7.76 & -16.25 & 0.71 & 0.07 \\
TSH & 14.28 & -12.66 & 41.27 & 0.28 \\
FT4 & -2.12 & -11.75 & 7.5 & 0.65 \\
Cosmetic & -0.117 & -3.39 & 0.16 & 0.94 \\
Symptom & 0.819 & -0.701 & 2.33 & 0.278 \\
Ablation time & 0.823 & -1.108 & 2.75 & 0.38 \\
Volume lidocaine 2\% & 0.506 & -2.38 & 3.39 & 0.72 \\
Min RF power & -0.385 & -1.57 & 0.806 & 0.511 \\
Max RF power & 0.016 & -1.57 & 1.61 & 0.98 \\
Largest diameter & -1.96 & -5.659 & 1.729 & 0.284 \\
Initial volume & & & & \\
\hline
\end{tabular}

limitations such as general anesthesia, iatrogenic hypothyroidism, scarring of the neck, atrial fibrillation, and increasing overall morbidity and mortality from cardiovascular diseases [6, 7]. Radiofrequency ablation has been adopted worldwide as a minimally invasive treatment of benign thyroid nodules. It has proven to be a safe and effective option, but the results have been heterogeneous in many studies. The VRR ranged from $32.7-58.2 \%$ at 1 month [22-24] and 50-85.5\% after 3 months depending on different ablation machine systems, basic techniques, and thyroid nodule's characteristics in the studies [25-27]. Our study showed suitability in VRR results during the 1month and 3-month follow-up, matched with the literature. A gradual progression of VRR, which was approximately $44.6-84.1 \%$ at 6 months; $58-89.6 \%$ at 12 months; and $84-88 \%$ at 2 years, was apparent in many longer follow-up studies $[25,28-33]$. The quick decrease of nodule volume within 3 months after ablation helps to improve the symptomatic and cosmetic problems.

The safety profile of thyroid RFA was demonstrated in the literature. In treating benign thyroid nodules, the prevalence of RFA complications was $2.11 \%$ for overall complications and $1.27 \%$ for major complications [34-36]. No major complications were found in our study and $2.56 \%$ of minor complications (transient voice change) occurred. It is all because of strictly applying two fundamental techniques (the moving-shot technique and the trans-isthmic approach) during the RFA procedure. These techniques help to not only prevent hot fluid escape and the electrode tip changing in the position when patients talk, swallow, or cough but also limiting damage to surrounding tissue $[12,19]$. Moreover, in a few cases to prevent important structures such as nerves and arteries when complete ablation of the nodule with unfavorable anatomy was desired, the hydrodissection technique was used by injecting slowly $5 \%$ dextrose. For management of voice change after RFA, we injected cold $5 \%$ dextrose $\left(0^{\circ} \mathrm{C}\right.$ to $\left.4^{\circ} \mathrm{C}\right)$ as the same hydrodissection technique [37]. In addition, our study showed the thyroid function test result was unchanged. These results agree with some study conclusions that the rate of hypothyroidism and hypoparathyroidism was reduced as compared to surgery.

Factors related to the efficacy of RFA therapy for benign thyroid nodule(s) have been reported differently in numerous studies. Some studies showed that the initial volume of the nodule seems to be a significant predictive factor of the RFA efficacy $[13-15,31]$. The solidity of the nodule is the significant factor that affected to the efficacy of this therapy in the other studies $[14,15,17]$. Jung et al. revealed that solidity and energy delivered were independent factors that predicted the final volume reduction [16]. In agreement with previous findings, our study confirmed the internal component of the nodules as an independent factor affecting the VRR. Higher VRR occurred in cystic or predominantly cystic nodules. The cystic fluid of the nodule was aspirated before the RFA procedure followed by a quick reduction in the volume of the ablated nodule. Also, because solid components and their vascularity which secrete the fluid were ablated, the recurrence of a cystic nodule was effectively prevented. However, our results showed no association between other factors (baseline characteristics of the patients and nodules and treatment characteristics) and the VRR. Recently, Sim et al. showed that the IAR is a factor highly correlated with the VRR. If the IAR is greater than $70 \%$ and the VRR is greater than $50 \%$, therapeutic success may be expected after RFA [18].

Our study has several limitations. Firstly, the follow-up in this prospective study was largely affected by irregular patient follow-up intervals during the COVID-19 pandemic. A single-center study with relatively small sample size and short time follow-up of 1 month and 3 months is another limitation. Some variables including vascularity of nodule and IAR were not collected. These limitations inspire our 
group to do larger and longer prospective multicenter cohort studies to prove these results.

In conclusion, RFA was demonstrated as a safe and effective option for benign thyroid nodule treatment. The VRR reached $41.47 \%$ and $64.72 \% 1$ and 3 months after ablation. Minor complication (transient voice change) occurred in two cases after the RFA procedure, and thyroid functions were constant. We found only the internal component of nodules as an independent factor affecting the VRR during the 3-month follow-up. Thus, RFA can be used as an alternative treatment with encouraging results and fewer complications.

\section{Abbreviations}

$\begin{array}{ll}\text { ACR- } & \text { American College of Radiology-Thyroid } \\ \text { TIRADS: } & \text { Imaging Reporting and Data Systems } \\ \text { CI: } & \text { Confidence interval } \\ \text { CNB: } & \text { Core needle biopsy } \\ \text { FNA: } & \text { Fine-needle aspiration } \\ \text { FT4: } & \text { Free thyroxine } \\ \text { IAR: } & \text { Initial ablation ratio } \\ \text { RF: } & \text { Radiofrequency } \\ \text { RFA: } & \text { Radiofrequency ablation } \\ \text { SD: } & \text { Standard deviation } \\ \text { TSH: } & \text { Thyrotropin } \\ \text { VRR: } & \text { Volume reduction rate } \\ \text { US: } & \text { Ultrasound. }\end{array}$

\section{Data Availability}

Data and materials supporting our findings will be shared upon request.

\section{Consent}

Written informed consent form was given to patients.

\section{Conflicts of Interest}

The authors declare no conflicts of interest.

\section{Authors' Contributions}

All authors contributed to data analysis and drafting or revising the article, have agreed on the journal to which the article will be submitted, gave final approval of the version to be published, and agreed to be accountable for all aspects of the work.

\section{Acknowledgments}

The authors would like to thank the patients who agreed to participate in this cohort study and Danang Family hospital for financial support.

\section{References}

[1] S. Ezzat, D. A. Sarti, D. R. Cain, and G. D. Braunstein, "Thyroid incidentalomas. Prevalence by palpation and ultrasonography," Archives of Internal Medicine, vol. 154, no. 16, pp. 1838-1840, 1994.

[2] A. Brander, P. Viikinkoski, J. Nickels, and L. Kivisaari, "Thyroid gland: US screening in a random adult population," Radiology, vol. 181, no. 3, pp. 683-687, 1991.

[3] P. Miccoli, M. N. Minuto, C. Ugolini, R. Pisano, A. Fosso, and P. Berti, "Minimally invasive video-assisted thyroidectomy for benign thyroid disease: an evidence-based review," World Journal of Surgery, vol. 32, no. 7, pp. 1333-1340, 2008.

[4] J. H. Kim, J. H. Baek, H. K. Lim et al., "2017 thyroid radiofrequency ablation guideline: korean society of thyroid radiology," Korean Journal of Radiology, vol. 19, no. 4, pp. 632-655, 2018.

[5] W.-W. Yue, S.-R. Wang, F. Lu et al., "Quality of life and costeffectiveness of radiofrequency ablation versus open surgery for benign thyroid nodules: a retrospective cohort study," Scientific Reports, vol. 6, no. 1, Article ID 37838, 2016.

[6] E. Bandeira-Echtler, K. Bergerhoff, and B. Richter, "Levothyroxine or minimally invasive therapies for benign thyroid nodules," Cochrane Database of Systematic Reviews, vol. 6, no. 6, Article ID Cd004098, 2014.

[7] B. R. Haugen, E. K. Alexander, K. C. Bible et al., "2015 American thyroid association management guidelines for adult patients with thyroid nodules and differentiated thyroid cancer: the American thyroid association guidelines Task Force on thyroid nodules and differentiated thyroid cancer," Thyroid, vol. 26, no. 1, pp. 1-133, 2016.

[8] E. J. Ha, J. H. Baek, and J. H. Lee, "The efficacy and complications of radiofrequency ablation of thyroid nodules," Current Opinion in Endocrinology andDiabetes and Obesity, vol. 18, no. 5, pp. 310-314, 2011.

[9] R. Cesareo, A. Palermo, V. Pasqualini et al., "Efficacy and safety of a single radiofrequency ablation of solid benign nonfunctioning thyroid nodules," Archives of Endocrinology and Metabolism, vol. 61, no. 2, pp. 173-179, 2017.

[10] Y. Che, S. Jin, C. Shi et al., "Treatment of benign thyroid nodules: comparison of surgery with radiofrequency ablation," American Journal of Neuroradiology, vol. 36, no. 7, pp. 1321-1325, 2015.

[11] J. H. Baek, J. H. Lee, R. Valcavi, C. M. Pacella, H. Rhim, and D. G. Na, "Thermal ablation for benign thyroid nodules: radiofrequency and laser," Korean Journal of Radiology, vol. 12, no. 5, pp. 525-540, 2011.

[12] H. S. Park, J. H. Baek, Y. J. Choi, and J. H. Lee, "Innovative techniques for image-guided ablation of benign thyroid nodules: combined ethanol and radiofrequency ablation," Korean Journal of Radiology, vol. 18, no. 3, pp. 461-469, 2017.

[13] R. Cesareo, A. M. Naciu, M. Iozzino et al., "Nodule size as predictive factor of efficacy of radiofrequency ablation in treating autonomously functioning thyroid nodules," International Journal of Hyperthermia, vol. 34, no. 5, pp. 617-623, 2018.

[14] M. Deandrea, F. Garino, M. Alberto et al., "Radiofrequency ablation for benign thyroid nodules according to different US features: an Italian multicentre prospective study," European Journal of Endocrinology, vol. 180, 2018.

[15] H. Dobnig and K. Amrein, "Monopolar radiofrequency ablation of thyroid nodules: a prospective Austrian single-center study," Thyroid, vol. 28, no. 4, pp. 472-480, 2018.

[16] S. L. Jung, J. H. Baek, J. H. Lee et al., "Efficacy and safety of radiofrequency ablation for benign thyroid nodules: a prospective multicenter study," Korean Journal of Radiology, vol. 19, no. 1, pp. 167-174, 2018. 
[17] G. M. Lee, J. Y. You, H. Y. Kim et al., "Successful radiofrequency ablation strategies for benign thyroid nodules," Endocrine, vol. 64, 2018.

[18] J. S. Sim, J. H. Baek, and W. Cho, "Initial ablation ratio: quantitative value predicting the therapeutic success of thyroid radiofrequency ablation," Thyroid, vol. 28, no. 11, pp. 1443-1449, 2018.

[19] E. J. Ha, J. H. Baek, Y. Che et al., "Radiofrequency ablation of benign thyroid nodules: recommendations from the asian conference on tumor ablation Task Force," Ultrasonography, vol. 40,2020

[20] J. H. Baek, Y. S. Kim, D. Lee, J. Y. Huh, and J. H. Lee, "Benign predominantly solid thyroid nodules: prospective study of efficacy of sonographically guided radiofrequency ablation versus control condition," American Journal of Roentgenology, vol. 194, no. 4, pp. 1137-1142, 2010.

[21] M. Ahmed, L. Solbiati, C. L. Brace et al., "Image-guided tumor ablation: standardization of terminology and reporting criteria-a 10 year update," Radiology, vol. 273, no. 1, pp. 241-260, 2014.

[22] W. K. Jeong, J. H. Baek, H. Rhim et al., "Radiofrequency ablation of benign thyroid nodules: safety and imaging followup in 236 patients," European Radiology, vol. 18, no. 6, pp. 1244-1250, 2008.

[23] J. Y. Sung, J. H. Baek, S. L. Jung et al., "Radiofrequency ablation for autonomously functioning thyroid nodules: a multicenter study," Thyroid, vol. 25, no. 1, pp. 112-117, 2015.

[24] M. Deandrea, P. Limone, E. Basso et al., "US-guided percutaneous radiofrequency thermal ablation for the treatment of solid benign hyperfunctioning or compressive thyroid nodules," Ultrasound in Medicine and Biology, vol. 34, no. 5, pp. 784-791, 2008.

[25] S. Bernardi, F. Stacul, A. Michelli et al., "12-month efficacy of a single radiofrequency ablation on autonomously functioning thyroid nodules," Endocrine, vol. 57, no. 3, pp. 402-408, 2017.

[26] K. D. Kohlhase, Y. Korkusuz, D. Gröner et al., "Bipolar radiofrequency ablation of benign thyroid nodules using a multiple overlapping shot technique in a 3-month follow-up," International Journal of Hyperthermia, vol. 32, no. 5, pp. 511-516, 2016.

[27] M. S. Abd El-Galil, A. H. Ali, R. M. Botros, Y. I. Abd ElKhaleq, and O. M. A. Hetta, "Efficacy and safety of ultrasound (US)-guided radiofrequency ablation of benign thyroid nodules," Egyptian Journal of Radiology and Nuclear Medicine, vol. 52, no. 1, p. 57, 2021.

[28] E. Aysan, U. O. Idiz, H. Akbulut, and L. Elmas, "Single-session radiofrequency ablation on benign thyroid nodules: a prospective single center study," Langenbeck's Archives of Surgery, vol. 401, no. 3, pp. 357-363, 2016.

[29] A. Doros, P. Reismann, G. Huszty et al., "Treatment of benign thyroid nodules by radiofrequency thermal ablation," Orvosi Hetilap, vol. 161, no. 27, pp. 1131-1136, 2020.

[30] O. Hamidi, M. R. Callstrom, R. A. Lee et al., "Outcomes of radiofrequency ablation therapy for large benign thyroid nodules: a mayo clinic case series," Mayo Clinic Proceedings, vol. 93, no. 8, pp. 1018-1025, 2018.

[31] F. Feroci, T. Guagni, A. Coppola et al., "Radiofrequency thermal ablation of benign thyroid nodules: the correlation between ultrasound nodule characteristics and results," Surgical Innovation, vol. 27, Article ID 1553350620913134, 2020.

[32] J. Aldea Martínez, L. Aldea Viana, J. L. López Martínez, and E. Ruiz Pérez, "Radiofrequency ablation of thyroid nodules: a long-term prospective study of 24 patients," Journal of
Vascular and Interventional Radiology, vol. 30, no. 10, pp. 1567-1573, 2019.

[33] Y. Guang, W. He, Y. Luo et al., "Patient satisfaction of radiofrequency ablation for symptomatic benign solid thyroid nodules: our experience for 2-year follow up," BMC Cancer, vol. 19, no. 1, p. 147, 2019.

[34] J.-F. Wang, T. Wu, K.-P. Hu et al., "Complications following radiofrequency ablation of benign thyroid nodules," Chinese Medical Journal, vol. 130, no. 11, pp. 1361-1370, 2017.

[35] J. H. Baek, J. H. Lee, J. Y. Sung et al., "Complications encountered in the treatment of benign thyroid nodules with US-guided radiofrequency ablation: a multicenter study," Radiology, vol. 262, no. 1, pp. 335-342, 2012.

[36] S. R. Chung, C. H. Suh, J. H. Baek, H. S. Park, Y. J. Choi, and J. H. Lee, "Safety of radiofrequency ablation of benign thyroid nodules and recurrent thyroid cancers: a systematic review and meta-analysis," International Journal of Hyperthermia: The Official Journal of European Society for Hyperthermic Oncology, North American Hyperthermia Group, vol. 33, no. 8, pp. 920-930, 2017.

[37] S. R. Chung, J. H. Baek, Y. J. Choi, and J. H. Lee, "Management strategy for nerve damage during radiofrequency ablation of thyroid nodules," International Journal of Hyperthermia, vol. 36, pp. 1-7, 2019. 